\title{
ON SEMIGROUPS WHOSE IDEMPOTENT-GENERATED SUBSEMIGROUP IS APERIODIC
}

\author{
MANUEL DELGADO \\ Centro de Matemática, Universidade do Porto \\ Rua do Campo Alegre 687, 4169-007 Porto, Portugal \\ mdelgado@fc.up.pt. \\ VÍTOR H. FERNANDES \\ Departamento de Matemática, Faculdade de Ciências e Tecnologia \\ Universidade Nova de Lisboa, Monte de Caparica, 2829-516 Caparica, Portugal \\ and \\ C.A.U.L., Av. Prof. Gama Pinto 2, 1649-003 Lisboa, Portugal \\ vhf@fct.unl.pt. \\ STUART MARGOLIS \\ Department of Mathematics, Bar Ilan University \\ 52900 Ramat Gan, Israel \\ stwm@math.huji.ac.il. \\ BENJAMIN STEINBERG \\ Carleton University, School of Mathematics \& Statistics \\ 1125 Colonel By Drive, Ottawa, Ontario K1S 5B6, Canada \\ bsteinbg@math.carleton.ca. \\ Received 2 February 2003 \\ Revised 18 December 2003 \\ Communicated by J. Meakin
}

We show that if $S$ is a finite semigroup with aperiodic idempotent-generated subsemigroup and $\mathbf{H}$ is a pseudovariety of groups, then the sequence of iterated $\mathbf{H}$-kernels of $S$ stops at the idempotent-generated subsemigroup if and only if each subgroup of $S$ belongs to the wreath product closure of $\mathbf{H}$. Applications are given to Mal'cev products.

Keywords: Iterated kernels; wreath product closure; Mal'cev products.

Mathematics Subject Classification 2000: 20M07, 20M35, 20M12

\section{Introduction}

In this paper, all semigroups are assumed to be finite unless otherwise stated. If $\mathbf{H}$ is a pseudovariety of groups (i.e. a class of groups closed under formation of finite 
direct products, subgroups and quotients groups), then it is natural to define a group to be $\mathbf{H}$-solvable if it has a subnormal series, each of whose quotients belongs to $\mathbf{H}$. For instance, a group is solvable in the classical sense if and only if it is $\mathbf{A b}$ solvable where $\mathbf{A b}$ is the pseudovariety of Abelian groups. A group is a $p$-group if and only if it is $\mathbf{Z}_{p}$-solvable where $\mathbf{Z}_{p}$ is the pseudovariety generated by a cyclic group of order $p$. One should not confuse $\mathbf{Z}_{p}$-solvablity with the classical notion of $p$-solvability [16]. Recall [9] that a group $G$ is $p$-solvable if it has a normal series each of whose quotients is a $p$-group or a $p^{\prime}$-group (the latter meaning the order is relatively prime to $p$ ). If $\mathbf{H}$ is the pseudovariety generated by $\mathbf{Z}_{p}$ and the simple $p^{\prime}$-groups, then the $p$-solvable groups are precisely the $\mathbf{H}$-solvable groups in our sense.

It is easy to see that a group is $\mathbf{H}$-solvable if and only if its simple group divisors belong to $\mathbf{H}$ and moreover the collection of $\mathbf{H}$-solvable groups is the smallest pseudovariety of groups containing $\mathbf{H}$ and closed under extension (or equivalently wreath product); see [6]. This pseudovariety is denoted $\mathcal{W H}$.

Alternatively a group is $\mathbf{H}$-solvable if iteration of the operation of taking the verbal subgroup corresponding to $\mathbf{H}$ eventually yields the trivial subgroup. For instance, a group is solvable if and only if iteration of the derived subgroup eventually arrives at the trivial subgroup.

One possible way to generalize these notions to semigroups is to consider all semigroups whose simple group divisors belong to $\mathbf{H}$. Equivalently, one is considering all semigroups whose subgroups belong to $\mathcal{W H}$. This set forms a pseudovariety of semigroups (defined analogously to the group case), which is normally denoted $\overline{\mathcal{W H}}$. The Krohn-Rhodes theorem [3,6] implies that this pseudovariety consists precisely of all semigroups that divide a wreath product of groups in $\mathbf{H}$ and copies of the monoid obtained by adjoining a unit to the two element right zero semigroup. However, we do not choose this as the definition of $\mathbf{H}$-solvability for semigroups. Instead we choose a definition introduced by the first and second authors in [5].

In [5], motivated by the fact that the verbal subgroup associated to $\mathbf{H}$ of a group can be generalized to semigroups via the notion of the $\mathbf{H}$-kernel, they defined a semigroup to be $\mathbf{H}$-solvable if iterating the $\mathbf{H}$-kernel operator eventually arrives at the subsemigroup generated by the idempotents. In [5], they showed that a semigroup with commuting idempotents is $\mathbf{A b}$-solvable if and only if its subgroups are solvable groups. This paper supersedes [5] by proving a much more general result that is in some sense optimal. This paper can be read independently of [5], although that paper is a source for several ideas in this paper.

For a pseudovariety $\mathbf{V}$ of semigroups, we denote by EV the pseudovariety consisting of all semigroups whose idempotent-generated subsemigroup belongs to $\mathbf{V}$.

Let $\mathbf{A}$ be the pseudovariety of all aperiodic semigroups and let $\mathbf{V}$ be a subpseudovariety of A. Recall that $(\mathrm{m}$ ) denotes the Mal'cev product. Our main result states that, for a non-trivial pseudovariety $\mathbf{H}$ of groups,

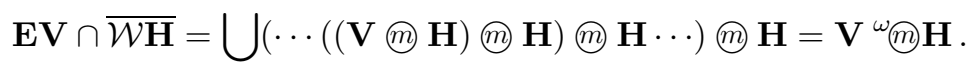


In particular,

$$
\mathbf{E A}=\mathbf{A}^{\omega} \mathbb{m} \mathbf{G}
$$

There are many examples of pseudovarieties $\mathbf{V} \subseteq \mathbf{A}$ such that the stronger equality $\mathbf{E V}=\mathbf{V}: \mathbf{G}$ holds. Most of them are direct consequences of the main result of [15]. Denote by $\operatorname{Reg}(S)$ the set of regular elements of $S$ and let DA be the pseudovariety consisting of all semigroups whose regular elements are idempotents. Let

$$
\mathbf{R V}=\{S \mid\langle\operatorname{Reg}(S)\rangle \in \mathbf{V}\}
$$

Results of [15] imply that $\operatorname{Reg}(\langle E(S)\rangle)=\operatorname{Reg}\left(\mathrm{K}_{\mathbf{G}}(S)\right)$, for $S \in \mathbf{E D A}$ (where $\mathrm{K}_{\mathbf{G}}(S)$ is the G-kernel of $S$ ). Hence, if $\mathbf{V} \subseteq \mathbf{D A}$ is such that $\mathbf{V}=\mathbf{R V}$, then

$$
\mathbf{E V}=\mathbf{V}: \mathbf{G} .
$$

Clearly $\mathbf{R V}=\mathbf{V}$ for $\mathbf{V}$ any of $\mathbf{J}, \mathbf{R}, \mathbf{L}$ or $\mathbf{D A}$, where $\mathbf{J}, \mathbf{R}$ and $\mathbf{L}$ are respectively the classes of all $\mathcal{J}$-trivial, $\mathcal{R}$-trivial, and $\mathcal{L}$-trivial semigroups. See [10] for more information on pseudovarieties of the form $\mathbf{V}: \mathbf{G}$. See also [2] for some new examples and syntactic proofs.

It is natural to ask: does $\mathbf{E A}=\mathbf{A}: \mathbf{m}$ G? The answer is negative. In fact, although all semigroups in $\mathbf{A}(\mathrm{m} \mathbf{G}$ have complexity 1, Rhodes and Tilson [15] have shown the existence of regular semigroups of arbitrary complexity in EA. The subpseudovariety $(\mathbf{A}: \mathbf{m} \mathbf{G})$ : $\mathbf{G}$ of EA also has semigroups of larger complexity. Rhodes has a published example [13] of a semigroup of complexity 2 in $(\mathbf{A}: \mathbf{G}): \mathbf{G}$ and has unpublished examples of semigroups with arbitrary complexity in this pseudovariety. A construction of Rhodes and Tilson [15] implies that, for all positive integers $n$ and any non-trivial pseudovariety $\mathbf{H}$ of groups, $\mathbf{A}^{n} m \mathbf{H} \neq \mathbf{A}^{n-1} m \mathbf{H}$. It follows that the hierarchy $\mathbf{E A} \cap \overline{\mathcal{W}} \mathbf{H}=\mathbf{A}^{\omega} m \mathbf{H}$ is strict, that is $\mathbf{E A} \cap \overline{\mathcal{W}} \mathbf{H} \neq \mathbf{A}^{n} m \mathbf{H}$, for any positive integer $n$.

\section{Definitions, Notation and Preliminaries}

For basic notions related to pseudovarieties of semigroups or monoids we refer the reader to Almeida's book [1]. For background on Green's relations and Rees matrix semigroups see the book of Clifford and Preston [4] and the chapters of Krohn, Rhodes and Tilson in [3]; see also Lallement's book [12] and Tilson's chapters in Eilenberg [6].

Let $S$ be a semigroup. As usual, denote by $S^{I}$ the monoid obtained from $S$ by adjoining an identity element and, for a subset $X$ of $S$, denote by $E(X)$ the subset of all idempotents of $S$ contained in $X$ and by $\langle X\rangle$ the subsemigroup of $S$ generated by $X$.

The quasi-order $\leq_{\mathcal{J}}$ associated to Green's relation $\mathcal{J}$ is defined by: for all $u, v \in S$,

$$
u \leq \mathfrak{g} v \quad \text { if and only if } \quad S^{I} u S^{I} \subseteq S^{I} v S^{I} .
$$


Of course, for every $u, v \in S, u \mathcal{J} v$ if and only if $u \leq_{\mathcal{J}} v$ and $v \leq_{\mathcal{J}} u$. Denote by $J_{u}$ the $\mathcal{J}$-class of the element $u \in S$. As usual, a partial order relation $\leq_{\mathrm{g}}$ is defined on the set $S / \mathcal{J}$ by setting, for all $u, v \in S, J_{u} \leq_{\mathfrak{J}} J_{v}$ if and only if $u \leq_{\mathfrak{g}} v$. Given $u, v \in S$, we write $u<_{\mathfrak{g}} v$ and $J_{u}<_{\mathfrak{J}} J_{v}$ if and only if $u \leq_{\mathfrak{g}} v$ and $(u, v) \notin \mathcal{J}$.

Similar notations are used for Green's relations $\mathcal{R}, \mathcal{L}, \mathcal{H}=\mathcal{R} \cap \mathcal{L}$ and $\mathcal{D}=\mathcal{R} \vee \mathcal{L}$.

Let $S$ be a semigroup and $J$ be a $\mathcal{J}$-class of $S$. We denote by $J^{0}$ the semigroup with support $J \cup\{0\}$, with $0 \notin J$, and multiplication defined by:

$$
a \cdot b= \begin{cases}a b & \text { if } a, b \in J \text { and } a b \in J \\ 0 & \text { otherwise }\end{cases}
$$

for all $a, b \in J \cup\{0\}$.

Now, let $A$ and $B$ be non-empty sets, $G$ a group and $C: B \times A \rightarrow G^{0}$ a $B \times A$ matrix over $G^{0}$. The Rees matrix semigroup $\mathcal{M}^{0}(G, A, B, C)$ is the semigroup with support $(A \times G \times B) \cup\{0\}$ and multiplication defined by

$$
(a, g, b) 0=0(a, g, b)=00=0
$$

and

$$
(a, g, b)\left(a^{\prime}, g^{\prime}, b^{\prime}\right)= \begin{cases}\left(a, g C\left(b, a^{\prime}\right) g^{\prime}, b^{\prime}\right) & \text { if } C\left(b, a^{\prime}\right) \in G \\ 0 & \text { otherwise (i.e. } \left.C\left(b, a^{\prime}\right)=0\right),\end{cases}
$$

for all $a, a^{\prime} \in A, b, b^{\prime} \in B$ and $g, g^{\prime} \in G$.

It is well-known that $\mathcal{M}^{0}(G, A, B, C)$ is a regular semigroup if and only if $C$ is a regular matrix, i.e. each row and column of $C$ has at least one non-zero entry, and in this case $\mathcal{M}^{0}(G, A, B, C)$ is a (completely) 0-simple semigroup.

Given semigroups $S$ and $T$, a relational morphism of semigroups $\tau: S \multimap T$ is a function from $S$ into $\mathcal{P}(T)$, such that $\tau\left(s_{1}\right) \neq \emptyset$ and $\tau\left(s_{1}\right) \tau\left(s_{2}\right) \subseteq \tau\left(s_{1} s_{2}\right)$, for all $s_{1}, s_{2} \in S$.

A relational morphism $\tau: S \nrightarrow T$ is, in particular, a relation from $S$ to $T$ and we may compose relational morphisms in the obvious way. Homomorphisms, viewed as relations, and inverses of onto homomorphisms are examples of relational morphisms.

From now on, $\mathbf{H}$ will always denote a pseudovariety of groups; we use $\overline{\mathbf{H}}$ for the pseudovariety of semigroups whose subgroups are in $\mathbf{H}$.

The H-kernel of a semigroup $S$ is the subsemigroup $\mathrm{K}_{\mathbf{H}}(S)=\bigcap \tau^{-1}(1)$, with the intersection being taken over all relational morphisms $\tau: S \multimap G$, with $G \in \mathbf{H}$. It is well-known [15] that there always exists a relational morphism $\tau: S \rightarrow G$, with $G \in \mathbf{H}$ such that $\tau^{-1}(1)=\mathrm{K}_{\mathbf{H}}(S)$.

One of the most widely studied operators on the lattice of pseudovarieties is the Mal'cev product; see [14] for more on operators and iteration of operators on the lattice of pseudovarieties. Its definition, when the rightmost factor is a pseudovariety of groups, may be formulated as follows: for a pseudovariety $\mathbf{V}$, the Mal'cev product of $\mathbf{V}$ and $\mathbf{H}$ is the pseudovariety

$$
\mathbf{V}\left(m \mathbf{H}=\left\{S \mid K_{\mathbf{H}}(S) \in \mathbf{V}\right\} .\right.
$$


We refer the reader to [10] for some history concerning the Mal'cev product and for connections with the all-important semidirect product.

Next, we define recursively $\mathrm{K}_{\mathbf{H}}^{(n)}(S)$ as follows:

- $\mathrm{K}_{\mathbf{H}}^{(0)}(S)=S$;

- $\mathrm{K}_{\mathbf{H}}^{(n)}(S)=\mathrm{K}_{\mathbf{H}}\left(\mathrm{K}_{\mathbf{H}}^{(n-1)}(S)\right)$, for $n \geq 1$.

Since $S$ is finite and the operator $\mathrm{K}_{\mathbf{H}}$ is non-increasing, it follows that the sequence $\mathrm{K}_{\mathbf{H}}^{(n)}(S)$ is eventually constant; we denote this constant value by $\mathrm{K}_{\mathbf{H}}^{(\omega)}(S)$. Observe that $\mathrm{K}_{\mathbf{H}}^{(\omega)}(S)$ is the largest subsemigroup of $S$ fixed by $\mathrm{K}_{\mathbf{H}}$.

We say that $S$ is $\mathbf{H}$-solvable if $\mathrm{K}_{\mathbf{H}}^{(\omega)}(S)=\langle E(S)\rangle$.

Herein, we consider iterated Mal'cev products. More specifically, we consider iterations of the operator (_) $m \mathbf{H}$. For a pseudovariety $\mathbf{V}$ and $n \geq 1$, we define the operator (_) ${ }^{n} m \mathbf{H}$ recursively as follows:

- $\mathbf{V}^{1}(\mathrm{~m} \mathbf{H}=\mathbf{V}: \mathbf{H}$;

- $\mathbf{V}^{n+1} m \mathbf{H}=\left(\mathbf{V}^{n}(\mathrm{~m}) \mathbf{H}\right) \mathbf{H}$;

- $\mathbf{V}^{\omega}(m) \mathbf{H}=\cup_{n \geq 1} \mathbf{V}^{n} m \mathbf{H}$.

We use left exponentiation to distinguish iteration of the operator (_) $\mathbb{m} \mathbf{H}$ from iteration of the operator $\mathbf{V}:(\mathbf{m})$.

It is easy to see that we have $\mathbf{V}^{n} m \mathbf{H}=\left\{S \mid \mathrm{K}_{\mathbf{H}}^{(n)}(S) \in \mathbf{V}\right\}$.

\section{The Main Result}

In this section, $\mathbf{H}$ denotes a non-trivial pseudovariety of groups.

First we state some preliminary results. The following lemma concerning the idempotent-generated subsemigroup of a semigroup is due to Fitzgerald [7].

Lemma 3.1. Let $S$ be a (perhaps infinite) semigroup and let $s \in\langle E(S)\rangle$ be an element of a regular $\mathcal{D}$-class $D$. Then, there exist $e_{1}, e_{2}, \ldots, e_{m} \in E(D)$ such that

$$
e_{1} \mathcal{R} e_{2} \mathcal{L} e_{3} \mathcal{R} \cdots \mathcal{L} e_{m-1} \mathcal{R} e_{m}
$$

and $s=e_{1} e_{2} \cdots e_{m}$. Hence $\langle E(S)\rangle \cap D=\langle E(D)\rangle \cap D$.

Recall that a $\mathcal{J}$-class is called null [3] if it is not regular.

Lemma 3.2. Let $S$ be a semigroup. If $J$ is a null J-class of $S$ such that $J \nsubseteq\langle E(S)\rangle$ and $J^{\prime}>_{\mathfrak{g}} J$ implies $J^{\prime} \subseteq\langle E(S)\rangle$, then $J \cap\langle E(S)\rangle=\emptyset$.

Proof. Suppose that there exists $t \in J \cap\langle E(S)\rangle$. If $s \in J$, then $s=x t y$, for some $x, y \in S^{I}$. As $J$ is null, we must have $x, y$ strictly $\mathcal{J}$-above $J$. Thus $x, y \in\langle E(S)\rangle^{I}$, which implies that $s \in\langle E(S)\rangle$, and therefore $J \subseteq\langle E(S)\rangle$, a contradiction.

Lemma 3.3. If $S \in \mathbf{E A}$ is $\mathbf{H}$-solvable, then the subgroups of $S$ are $\mathbf{H}$-solvable. 
Proof. Let $G$ be a subgroup of $S$. Then $\mathrm{K}_{\mathbf{H}}^{(\omega)}(G) \subseteq \mathrm{K}_{\mathbf{H}}^{(\omega)}(S)=\langle E(S)\rangle$. As $S \in \mathbf{E A}$, the semigroup $\langle E(S)\rangle$ is aperiodic and so its subgroup $\mathrm{K}_{\mathbf{H}}^{(\omega)}(G)$ must be trivial, as required.

In the sequel we shall make extensive use of the following theorem of Graham $[8]$.

Theorem 3.4. Let $S$ be a semigroup and let $J$ be a regular J-class of $S$. Then there exists an isomorphism (i.e. a Rees coordinatization)

$$
\iota: J^{0} \rightarrow \mathcal{M}^{0}(G, A, B, C)
$$

from $J^{0}$ to a Rees matrix semigroup $\mathcal{M}^{0}(G, A, B, C)$ such that:

- $A$ and $B$ can be partitioned into subsets $A_{1}, \ldots, A_{n}$ and $B_{1}, \ldots, B_{n}$, respectively.

- The matrix $C$ is the direct sum of the $n$ blocks $C_{1}, \ldots, C_{n}$, as suggested by the following picture (all the entries outside these blocks are zeros):

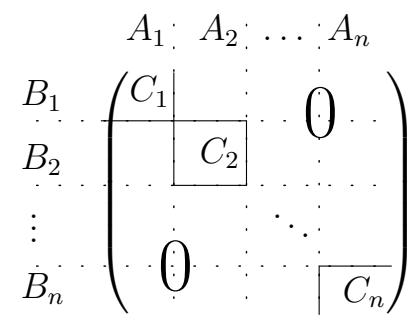

- Each matrix $C_{i}: B_{i} \times A_{i} \rightarrow G^{0}$ is regular and

$$
\left\langle E\left(J^{0}\right)\right\rangle=\cup_{i=1}^{n} \mathcal{M}^{0}\left(G_{i}, A_{i}, B_{i}, C_{i}\right),
$$

where $G_{i}$ is the subgroup of $G$ generated by all non-zero entries of $C_{i}$, for $i=$ $1, \ldots, n$.

A Rees coordinatization satisfying the properties described in the above theorem is called a Graham normalization of $J$ and can be computed in polynomial time from the multiplication table for $J^{0}[8]$.

Lemma 3.1 and Theorem 3.4 then combine to give the following result characterizing membership in EA.

Corollary 3.5. A semigroup $S$ belongs to EA if and only if, for each regular $\mathcal{J}$ class $J$, there exists a Rees coordinatization such that all the matrix entries are 0 or 1 if and only if, for each Graham normalization of such a J-class $J$, all the entries of the matrix are 0 or 1 .

Now, we are in a position to establish our main result. 
Theorem 3.6. Let $\mathbf{H}$ be a non-trivial pseudovariety of groups. Then every semigroup in $\mathbf{E A} \cap \overline{\mathcal{W H}}$ is $\mathbf{H}$-solvable.

Proof. Let $S \in \mathbf{E A} \cap \overline{\mathcal{W H}}$. The proof is by induction on $|S \backslash\langle E(S)\rangle|$. Clearly, the statement is true when $S=\langle E(S)\rangle$. Suppose now that $S \neq\langle E(S)\rangle$. Then, it suffices to construct a relational morphism $\tau: S \nrightarrow G \in \mathbf{H}$ such that $\left|\tau^{-1}(1)\right|<|S|$. Indeed, this would imply

$$
\left|\mathrm{K}_{\mathbf{H}}(S)\right| \leq\left|\tau^{-1}(1)\right|<|S|
$$

and so

$$
\left|\mathrm{K}_{\mathbf{H}}(S) \backslash\left\langle E\left(\mathrm{~K}_{\mathbf{H}}(S)\right)\right\rangle\right|<\left|S \backslash\left\langle E\left(\mathrm{~K}_{\mathbf{H}}(S)\right)\right\rangle\right|=|S \backslash\langle E(S)\rangle| .
$$

Hence, by induction,

$$
\mathrm{K}_{\mathbf{H}}^{(\omega)}\left(\mathrm{K}_{\mathbf{H}}(S)\right)=\left\langle E\left(\mathrm{~K}_{\mathbf{H}}(S)\right)\right\rangle
$$

that is,

$$
\mathrm{K}_{\mathbf{H}}^{(\omega)}(S)=\langle E(S)\rangle
$$

Let $J$ be a $\mathcal{J}$-class of $S$ which is $\leq_{\mathcal{J}}$-maximal amongst the $\mathcal{J}$-classes of $S$ not contained in $\langle E(S)\rangle$. To prove our result it suffices to construct a relational morphism $\tau: S \multimap H$ such that $\left|\tau^{-1}(1) \cap J\right|<|J|$. Consider the subsets $T(J)=\left\{s \in S \mid J<_{\mathfrak{J}} J_{s}\right\}$ and $B(J)=S \backslash T(J)$ of $S$. Figure 1 may help the reader to visualize the situation. Note that both $B(J)$ and $B(J) \backslash J$ are ideals of $S$. Two cases may arise: $J$ may be regular or null.

If $J$ is null, then by Lemma 3.2, $J \cap\langle E(S)\rangle=\emptyset$. It is then straightforward to verify that, for any non-trivial group $H \in \mathbf{H}$ and any fixed element $g \in H \backslash\{1\}$, the relation $\tau: S \longrightarrow H$ defined by

$$
\tau(x)= \begin{cases}\{1\} & \text { if } x \in T(J) \\ \{g\} & \text { if } x \in J \\ H & \text { otherwise (i.e. if } x \in B(J) \backslash J),\end{cases}
$$

for all $x \in S$, is a relational morphism such that $0=\left|\tau^{-1}(1) \cap J\right|<|J|$.

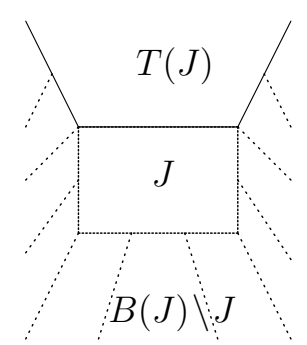

Fig. 1. 
Thus, it remains to consider the case where $J$ is regular. Let us consider a Graham normalization $\iota: J^{0} \rightarrow \mathcal{M}^{0}(G, A, B, C)$ of $J$ and suppose that $C$ is the direct sum of blocks $C_{1}, \ldots, C_{n}$, as in Graham's theorem. Without loss of generality we take $A$ and $B$ to be disjoint. Let $N=\mathrm{K}_{\mathbf{H}}(G)$. Since $\mathbf{H}$ is non-trivial, there is a prime number $p$ such that the cyclic group $\mathbb{Z}_{p}$ belongs to $\mathbf{H}$. We may then consider the vector space $\mathbb{Z}_{p}^{n}$ over $\mathbb{Z}_{p}$ with base $\left\{v_{1}, \ldots, v_{n}\right\}$. The construction of the required relational morphism is divided into several steps.

Step 1. First, we construct a partial homomorphism $\varphi: J \rightarrow G / N \times \mathbb{Z}_{p}^{n}$. Recall that $\varphi$ is a partial homomorphism if

$$
s, t, s t \in J \Rightarrow \varphi(s) \varphi(t)=\varphi(s t) .
$$

To this effect we consider the auxiliary function bl : $A \cup B \rightarrow\left\{v_{1}, \ldots, v_{n}\right\}$ defined by $\mathrm{bl}(x)=v_{i}$, if $x \in A_{i} \cup B_{i}$. Then, we define $\varphi$ by $\varphi(a, g, b)=(N g$, bl $(a)-\mathrm{bl}(b))$, for all $(a, g, b) \in J$. Next, we prove that $\varphi$ is, indeed, a partial homomorphism. First, we observe that $(a, g, b)\left(a^{\prime}, g^{\prime}, b^{\prime}\right) \in J$ if and only if $C\left(b, a^{\prime}\right) \neq 0$ (i.e. $C\left(b, a^{\prime}\right)=1$, by Corollary 3.5). Since $C$ is the direct sum of the $C_{i}$, from $C\left(b, a^{\prime}\right)=1$ it follows that $\mathrm{bl}(b)=\mathrm{bl}\left(a^{\prime}\right)$ and $\left(a, g g^{\prime}, b^{\prime}\right)=(a, g, b)\left(a^{\prime}, g^{\prime}, b^{\prime}\right)$. Thus

$$
\begin{aligned}
\varphi(a, g, b) \varphi\left(a^{\prime}, g^{\prime}, b^{\prime}\right) & =(N g, \mathrm{bl}(a)-\mathrm{bl}(b))\left(N g^{\prime}, \mathrm{bl}\left(a^{\prime}\right)-\mathrm{bl}\left(b^{\prime}\right)\right) \\
& =\left(N g g^{\prime}, \mathrm{bl}(a)-\mathrm{bl}(b)+\mathrm{bl}\left(a^{\prime}\right)-\mathrm{bl}\left(b^{\prime}\right)\right) \\
& =\left(N g g^{\prime}, \mathrm{bl}(a)-\mathrm{bl}\left(b^{\prime}\right)\right) \\
& =\varphi\left(a, g g^{\prime}, b^{\prime}\right) \\
& =\varphi\left((a, g, b)\left(a^{\prime}, g^{\prime}, b^{\prime}\right)\right)
\end{aligned}
$$

Note that, for an idempotent $e$, we have

$$
\varphi(e)=\varphi(e e)=\varphi(e) \varphi(e),
$$

whence $\varphi(e)=1=(N, 0)$. It follows that if $s \in\langle E(J)\rangle \cap J$ then $\varphi(s)=(N, 0)$.

Step 2. Next, we extend $\varphi$ to a relational morphism $\tau: B(J) \multimap G / N \times \mathbb{Z}_{p}^{n}$ by defining $\tau(s)=G / N \times \mathbb{Z}_{p}^{n}$, for all $s \in B(J) \backslash J$. Since $B(J) \backslash J$ is an ideal, $\tau$ is clearly a relational morphism.

Step 3. Finally, we extend $\tau$ to a relational morphism $\tilde{\tau}: S \multimap G / N \times \mathbb{Z}_{p}^{n}$ by defining $\tau(s)=\{1\}$, for all $s \in T(J)$.

In order to verify that $\tilde{\tau}$ is in fact a relational morphism, choose $s, t \in S$. Let us prove that $\tilde{\tau}(s) \tilde{\tau}(t) \subseteq \tilde{\tau}(s t)$. Since $\tau$ is a relational morphism and $B(J) \backslash J$ is an ideal, the only non-immediate cases are: $s, t \in T(J) ; s \in T(J)$ and $t \in J$; and $t \in T(J)$ and $s \in J$. First, let $s, t \in T(J)$. Then $\tilde{\tau}(s) \tilde{\tau}(t)=\{1\}$. If $s t \in T(J)$ or $s t \in B(J) \backslash J$, then clearly $1 \in \tilde{\tau}(s t)$. On the other hand, noting that $s, t \in\langle E(S)\rangle$, by the maximality 
of $J$, we conclude that if $s t \in J$, then $s t \in\langle E(S)\rangle \cap J=\langle E(J)\rangle \cap J$, by Lemma 3.1. Thus $\tilde{\tau}(s t)=\varphi(s t)=1$ and, in both cases, we have $\tilde{\tau}(s) \tilde{\tau}(t) \subseteq \tilde{\tau}(s t)$.

Next, consider $s \in T(J)$ and $t \in J$. Then st $\in B(J) \backslash J$ or $s t \in J$. In the first case, it is obvious that $\tilde{\tau}(s) \tilde{\tau}(t) \subseteq \tilde{\tau}(s t)$. So, suppose that $s t \in J$. Then, we will prove that $\varphi(s t)=\varphi(t)$. Let $t^{\prime}$ be an inverse of $t$. Then

$$
s t=s t t^{\prime} t=\left(s t t^{\prime}\right) t .
$$

Since $s \in\langle E(S)\rangle$ and $t t^{\prime} \in E(S)$, we have

$$
s t t^{\prime} \in\langle E(S)\rangle \cap J=\langle E(J)\rangle \cap J,
$$

by Lemma 3.1. Thus $\varphi\left(s t t^{\prime}\right)=1$. Therefore, $\varphi(s t)=\varphi\left(s t t^{\prime}\right) \varphi(t)=\varphi(t)$.

Finally, the case $t \in T(J)$ and $s \in J$ is dual to the previous one and so $\tilde{\tau}$ is, indeed, a relational morphism.

Now, one immediately sees from the definition that

$$
\tilde{\tau}^{-1}(N, 0) \cap J=\{(a, g, b) \mid \mathrm{bl}(a)=\mathrm{bl}(b) \text { and } g \in N\}
$$

and so we must consider two cases. First, if $J$ has more than one block, then

$$
\left|\tilde{\tau}^{-1}(N, 0) \cap J\right|<|J|,
$$

as required. On the other hand, if $J$ has only one block, then as $J \nsubseteq\langle E(S)\rangle$, by Graham's theorem, we must have that $G$ is non-trivial. Therefore, since $G \in \mathcal{W} \mathbf{H}$ (whence $\mathrm{K}_{\mathbf{H}}^{(\omega)}(G)=1$ ), we may conclude that $N=\mathrm{K}_{\mathbf{H}}(G)$ is a proper subgroup and so $\tilde{\tau}^{-1}(N, 0) \cap J$ is again strictly contained in $J$, completing the proof.

We remark that this proof is effective (assuming the decidability of $\mathbf{H}$ ) in the sense that we have explicitly constructed relational morphisms to groups in $\mathbf{H}$ witnessing that $\mathrm{K}_{\mathbf{H}}^{(\omega)}(S)=\langle E(S)\rangle$. Moreover, no computability hypotheses on $\mathrm{K}_{\mathbf{H}}$ were necessary.

Lemma 3.3, Theorem 3.6 and the fact that $\mathbf{V}{ }^{\omega} \rightarrow \mathbf{H} \subseteq \mathbf{E V}$ (see [5, 10]) imply the following result:

Corollary 3.7. Let $\mathbf{V} \subseteq \mathbf{A}$ and $1 \subsetneq \mathbf{H} \subseteq \mathbf{G}$. Then

$$
\mathbf{E V} \cap \overline{\mathcal{W H}}=\mathbf{V}^{\omega_{m}} \mathbf{H} .
$$

In particular, for $\mathbf{V}=\mathbf{S} \mathbf{l}$ and $\mathbf{H}=\mathbf{A b}$, we recover the main result from [5], where $\mathbf{G}_{\text {sol }}$ denotes the pseudovariety of all solvable finite groups.

Corollary 3.8. $\mathbf{E S l} \cap \overline{\mathbf{G}_{\text {sol }}}=\mathbf{S l}{ }^{\omega}(\mathrm{m} \mathbf{A b}=(\cdots((\mathbf{S l} ; \mathbf{A b})(m \mathbf{A b}) \cdots)$ m $\mathbf{A b}$.

Notice if one shifts to the right all the parentheses in the above corollary one obtains the strictly smaller pseudovariety $\mathbf{S l}: \mathbf{G}_{\text {sol }}[11]$. However it is still open whether an inverse semigroup belongs to $\mathbf{S l}: \mathbf{G}_{\text {sol }}$ if and only if its subgroups are solvable.

For $\mathbf{V}=\mathbf{A}$ and $\mathbf{H}=\mathbf{G}$, we obtain: 


\section{Corollary 3.9. $\mathbf{E A}=\mathbf{A}^{\omega} \mathrm{m} / \mathbf{G}$.}

Notice that, if $S \notin \mathbf{E A}$, then we cannot in general guarantee that $S \mathbf{H}$ solvable implies $S$ belongs to $\overline{\mathcal{W H}}$. In fact, for any positive integer $n$, the semigroup $\left\langle E\left(T_{n+1}\right)\right\rangle$, where $T_{n+1}$ is the full transformation semigroup on $n+1$ letters, is $\mathbf{H}$ solvable, but it contains the symmetric group $S_{n}$. It follows that unless $\mathbf{H}=\mathbf{G}$, there exists $\mathbf{H}$-solvable semigroups whose subgroups do not belong to $\overline{\mathcal{W H}}$.

\section{Acknowledgments}

The first and fourth author gratefully acknowledge support of FCT through CMUP and the FCT and POCTI Project POCTI/32817/MAT/2000 which is funded in cooperation with the European Community Fund FEDER.

The second author wishes to thank J.-E. Pin for facilitating his stay at LIAFA, University of Paris 7, where he was invited to stay between March and July, 2002. He gratefully acknowledges support of FCT and FEDER, within the project POCTI/32440/MAT/1999 - "Álgebra e Aplicações" of CAUL, and the fellowship SFRH/BSAB/244/2001.

The third author was partially supported by the Emmy Noether Research Institute for Mathematics and the Minerva Foundation of Germany and by the Excellency Center "Group Theoretic Methods in the study of Algebraic Varieties" of the Israel Science foundation; NSF DMS 9970471; BSF 1999298-1. He is also grateful for partial funding from the project POCTI/37670/MAT/2001.

The first, third and fourth authors were supported in part by INTAS 99-1224.

\section{References}

[1] J. Almeida, Finite Semigroups and Universal Algebra, English translation (World Scientific, Singapore, 1995).

[2] J. Almeida and A. Escada, On the equation $\mathbf{V} * \mathbf{G}=\mathbf{E V}$, J. Pure Appl. Algebra 166 (2002) $1-28$.

[3] M. Arbib, Algebraic Theory of Machines, Languages and Semigroups (Academic Press, New York, 1968).

[4] A. H. Clifford and G. B. Preston, The Algebraic Theory of Semigroups, Vol. I (American Mathematical Society, Providence, RI, 1961).

[5] M. Delgado and V. H. Fernandes, Solvable idempotent commuting monoids, to appear in Int. J. Algebra Computing.

[6] S. Eilenberg, Automata, Languages and Machines, Vol. B (Academic Press, New York, 1976).

[7] D. G. Fitzgerald, On inverses of products of idempotents in regular semigroups, $J$. Aust. Math. Soc. 13 (1972) 335-337.

[8] R. L. Graham, On finite 0-simple semigroups and graph theory, Math. Syst. Theory 2 (1968) 325-339.

[9] M. Hall, The Theory of Groups (AMS Chelsea Publishing, Providence, 1976).

[10] K. Henckell, S. Margolis, J.-E. Pin, and J. Rhodes, Ash's type II theorem, profinite topology and Malcev products, Part I, Int. J. Algebra Comput. 1 (1991) 411-436.

[11] P. Higgins and S. W. Margolis, Finite aperiodic semigroups with commuting idempotents and generalizations, Israel J. Math. 116 (2000) 367-380. 
[12] G. Lallement, Semigroups and Combinatorial Applications (Wiley, New York, 1979).

[13] J. Rhodes, Kernel systems - A global study of homomorphisms on finite semigroups, J. Algebra 49 (1977) 1-45.

[14] J. Rhodes and B. Steinberg, The $q$-theory of finite semigroups, preprint.

[15] J. Rhodes and B. Tilson, Improved lower bounds for the complexity of finite semigroups, J. Pure Appl. Algebra 2 (1972) 13-71.

[16] B. Tilson, Appendix to algebraic theory of finite semigroups: On the $p$-length of p-solvable semigroups, in Preliminary Results in Semigroups, ed. K. W. Folley (Academic Press, New York, London, 1969), pp. 163-208. 